\title{
Insertion Et Maintien Des Femmes Dans Les Groupements Associatifs En Côte d'Ivoire : Cas De La Coopérative COMAGOA-ROXY Dans La Commune d'Adjamé
}

\author{
Kouadio Amani Augustin \\ Yao Rosamonde \\ Mouroufie Kouassi Kouman Vincent \\ Laboratoire d'Etudes et \\ de Recherches Interdisciplinaires en Sciences Sociales \\ Institut d'Ethno-Sociologie, \\ Université Félix Houphouët-Boigny d'Abidjan, Cote d'Ivoire
}

Doi:10.19044/esj.2018.v14n35p258 URL:http://dx.doi.org/10.19044/esj.2018.v14n35p258

\begin{abstract}
This paper focuses on analyzing the strategies put in place by women to integrate and to maintain themselves in the activities of sale within the cooperative. In doing so, we rely, on the one hand, on the qualitative approach through the individual interviews with the Co-operative Comagoa ROXY at the Gouro market and the town hall of Adjamé. This was together with a focus group that brought together the few women members of the Cooperative. On the other hand, it was done using a quantitative approach through a questionnaire to co-operators of Comagoa ROXI. In total, the study revealed the mobilization by women of social capital as a strategy of insertion and maintenance in the cooperative. In the same way, the financial power of these women allows them to gain privileged positions while guaranteeing their maintenance within the cooperative.
\end{abstract}

Keywords: Cooperative, insertion, maintenance, social resources, Adjamé

Résumé

Cette étude se propose d'analyser les stratégies mises en place par les femmes pour s'intégrer et se maintenir dans les activités de vente au sein de la coopérative. Pour être mise en place, elle s'appuied'une part sur l'approche qualitative à travers les entretiens individuels avec les responsables de la coopérative COMAGOA ROXY au marché Gouro et de la mairie d'Adjamé et un groupe focus group qui a rassemblé les quelques femmes membres de la 
coopérative, et d'autre part sur l'approche quantitative à travers un questionnaire adressé aux coopératrices de COMAGOA ROXI. Au total, l'étude révèle l'intérêt pour les femmes d'intégrer le secteur informel, en particulier les groupements associatifs. Cela se matérialise par la mobilisation des femmes du capital social comme stratégie d'insertion et de maintien dans la coopérative. De même, la puissance financière de ces femmes leur permet d'acquérir une position privilégiée tout en garantissant leur maintien au sein de la coopérative.

Mots-clés : Coopérative, Insertion, Maintien, Ressources Sociales, Adjamé

\section{INTRODUCTION}

Le secteur informel regroupe un ensemble d'activités multiformes allant de la micro-entreprise (exemple de la vendeuse de vivriers) aux petites et moyennes entreprises en passant par les petites unités de production de biens ou de services. Ce secteur est l'un des piliers dynamiques des pays en voie de développement et sa progression annuelle est estimée à 7\%. Le secteur informel plus implicite dans les années 1970 a pris de l'ampleur au point de concurrencer le secteur formel. Les activités dites informelles sont un ensemble de stratégies trouvées par les populations pour surmonter les difficultés économiques et sociales liées aux politiques d'ajustements structurelles avec ses effets tels que la réduction des salaires, la diminution des effectifs de la fonction publique, la privatisation des entreprises, la cherté de la vie.... Cela a contribué à la dévalorisation du secteur public entraînant ainsi une augmentation du nombre d'agents opérant dans le secteur informel.

À cela, il faut ajouter une forte urbanisation qui fait des capitales africaines le théâtre d'une population active en expansion. Or, le secteur moderne ne peut pas offrir un nombre suffisant d'emplois à cette population. La stagnation du secteur moderne et des services entraine ainsi le développement du secteur informel qui lui offre une forte capacité de création d'emplois (Bacabar, 1993).

De cette manière, le secteur informel devient le « lieu de refuge » des personnes non scolarisées ou ayant un niveau scolaire peu élevé, des chômeurs et des personnes en quête d'emploi.

La problématique est que l'intégration dans les activités du secteur informel semble facile ; cependant, il s'avère que certaines structures œuvrant dans ce milieu, à savoir les coopératives, semble-t-il sont d'accès difficile et les personnes, pour s'y intégrer et se maintenir, vont devoir développer des stratégies.

Pour cette raison, cette étude se propose de comprendre les problèmes relatifs à l'insertion et au maintien des femmes dans les activités économiques au sein des groupements associatifs tels que la coopérative. 
L'objectif général de cet article est donc d'analyser les stratégies mises en place par les femmes pour s'insérer et se maintenir dans les activités de vente au sein de la coopérative COMAGOA ROXY et les logiques des coopératrices de COMAGOA-ROXI

\section{MÉTHODOLOGIE}

Cette étude à la fois qualitative et quantitative s'est réalisée dans la commune d'Adjamé, précisément au marché Gouro où se localise la coopérative COMAGOA ROXI. La collecte des données s'est faite sur une période d'un mois allant du 21 avril 2017 au 20 mai 2017, à travers l'échantillon par boule de neige dans les principes de la saturation des données pour l'approche qualitative et par l'échantillon de hasard simple dans l'approche quantitative. L'étude a pris en compte 10 femmes de la coopérative dans un entretien individuel et 10 femmes reparties en groupes de 5 au sein de deux groupes focus group.

Un guide d'entretien a été adressé également aux responsables de la coopérative, à savoir la présidente de la coopérative et le Directeur Général.

Dans l'approche quantitative un échantillon de 40 coopératrices a été utilisé à travers un questionnaire. Nous avons eu aussi à interroger les femmes qui vendent sur le site de la coopérative COMAGOA ROXY. Le choix des enquêtées n'a pas été fait au hasard. Elles ont été choisies en fonction de leur ancienneté sur le site ; c'est-à-dire, que ces femmes sont présentes sur le site de la COMAGOA depuis sa création. Nous avons tenu en compte ces femmes parce qu'elles font aussi le commerce du vivrier.

Nous avons afin adressé un guide d'entretien aux responsables de la mairie d'Adjamé.

L'analyse des résultats s'est fait à travers l'analyse stratégique de Michel Crozier selon laquelle chaque agent appartenant à un système mobilise des ressources pour accroître son pouvoir et atteindre son objectif.

Le récit de vie a également été utilisé comme un outil d'analyse privilégié de compréhension pour sonder le parcours existentiel des femmes au sein de la coopérative. C'est le récit des événements vécus par les femmes de la coopérative qui est appréhendé par cette technique.

\section{RÉSULTATS}

\section{Logique d'insertion et de maintien}

I-Caractéristique sociodémographique

\section{I.1 Situation matrimoniale}

Trois situations se présentent à ce niveau comme indiquées dans le tableau suivant. 
Tableau 1. Situation matrimoniale des coopératrices de COMAGOA ROXI

\begin{tabular}{|c|c|c|}
\hline & Valeurs absolues & Valeurs relatives en \% \\
\hline Mariées & 2 & 10 \\
\hline Célibataires & 4 & 85 \\
\hline Veuves & 34 & 100 \\
\hline Total & 40 & \\
\hline
\end{tabular}

Source : Notre enquête

Les coopératrices de COMAGOA ROXI sont majoritairement des femmes veuves. Elles représentent $85 \%$ des femmes. Cette forte proportion des veuves au sein de la coopérative s'explique par la vulnérabilité de ces dernières.

En effet, après le décès de leurs époux, les femmes se retrouvent seules à élever leurs enfants. Cette situation les plonge dans une certaine vulnérabilité. La recherche de soutien les conduit à la coopérative auprès d'une sœur ou d'une devancière. La coopérative devient ainsi, le lieu privilégié pour la recherche du réconfort et du soutien. L'exercice de cette activité qui consiste en la vente de produits vivriers leur permet désormais de se prendre en charge de même que leurs enfants et d'être économiquement autonomes.

À ce propos ALP, une des responsables affirme :

«mon mari là quand il vivait, je vendais un peu un peu mais depuis que il est mort là, je fais vivrier là bien même comme ça j'ai gagné l'argent pour me occuper de mon s'enfants, l'enfants de mes frères aussi sont avec moi. Où je suis là, vivrier c'est mon tout qui est là là. »

Outre les veuves, celles qui avaient des conjoints, avaient pour objectif le soutien de ces derniersà travers leur activité de vente pour les charges du foyer. Ainsi, les femmes considèrent la commercialisation du vivrier non pas une activité passagère mais comme une véritable profession à laquelle elles doivent leur survie.

\section{I.2 L'analphabétisme des femmes}

La majorité des femmes à COMAGOA ROXI est analphabète comme le témoigne le tableau suivant.

Tableau 2. Répartition des enquêtés selon le niveau d'instruction

\begin{tabular}{|c|c|c|}
\hline Niveau d'instruction & Valeur absolue & Valeur relative \\
\hline Aucun & 35 & $87.5 \%$ \\
\hline Primaire & 3 & $7.5 \%$ \\
\hline Secondaire & 2 & $5 \%$ \\
\hline Supérieur & 0 & $0 \%$ \\
\hline Total & 40 & $100 \%$ \\
\hline
\end{tabular}

Sources : Notre enquête

Il ressort au regard de ces résultats qu' environ 95\% des femmes de la coopérative n'ont pas dépassé le cycle primaire. Pour l'exercice de la 
commercialisation des produits vivriers, les femmes estiment qu'elles n'ont pas besoin d'instruction pour la réaliser. Ainsi, plusieurs d'entre elles ont commencé la commercialisation du vivrier depuis leur adolescence. C'est ce que dit NDK quand elle affirme ceci :

« Moi je ne suis pas allé à l'école donc c'est travail ça là que je peux faire. Depuis que je suis petite, ma maman m'a mise dans travail ça là, c'est ce que je connais et que je sais faire. Moi-même je ne comprends pas bien français, je me débrouille un peu un peu »

Eu égard à ces propos nous pouvons dire que la majorité de ces femmes n'ont pas été scolarisées et cela est renchérie par le Directeur Général de la COMAGOA qui affirme : «le problème d'analphabétisme de membres de la COMAGOA cause des disfonctionnements dans l'application des règles conformément à la loi qui régit les coopératives ».

Ce fait est corroboré par Azata Ouattara (2013), qui affirme que les femmes des marchés Gouro sont pour la plupart analphabètes ; pourtant, cela ne constitue pas un handicap pour elles. Au contraire, ayant pris conscience de leur rôle dans le développement économique de la Côte d'Ivoire, elles ont éprouvé le besoin de pérenniser leurs activités et de faire montre de leur savoirfaire en fournissant les marchés abidjanais en produits vivriers. Ainsi, malgré le fait de la non scolarisation, ces femmes se sentent importantes et elles ont leur place au sein de la société.

L'analphabétisme au sein des femmes de la coopérative COMAGOA peut s'expliquer par la pauvreté des parents d'une part et par leur appartenance ethnique d'autre part. En effet, la majorité des femmes sont de l'ethnie Gouro. Cette ethnie est reconnue pour sa grande production du vivrier en Côte d'Ivoire. Mais en plus de la production, les femmes Gouro se chargent de la commercialisation de ces vivriers. Dans la production du vivrier, elles ont pour habitude de travailler en association. C'est donc cette association des femmes dans la production qui a été transférée dans la commercialisation du vivrier à travers la coopérative COMAGOA. La commercialisation du vivrier est donc devenue l'activité principale des femmes Gouro, ce qui facilite leur insertion à la coopérative. Ne connaissant que cette activité, elles développent des stratégies pour être incontournables dans leur domaine. La maîtrise de cette activité, leur permet de s'y maintenir.

\section{Mobilisation des ressources comme stratégie d'intégration II.1 Appartenance ethnique}

La première stratégie utilisée par les femmes pour s'insérer dans coopérative COMAGOA-ROXI est l'appartenance ethnique. Pour entrer à la coopérative, les femmes brandissent d'abord leur appartenance à l'ethnie Gouro, ce qui fait que la majorité des femmes de la coopérative COMAGOA soient de cette ethnie. Selon la directrice, il y a environ 40 coopératrices et au 
moins 36 sont de l'ethnie Gouro. Les autres femmes autour des 4 restantes ont été introduites à la coopérative par leur camarade Gouro qui les a recommandées auprès des responsables de la coopérative.

« moi ça n'a pas été compliqué pour entrer dans la coopérative ma maman est Gouro et appartenait à la coopérative c'est elle qui m'a mise dedans ma maman nous a élevés dans vivrier là c'est ça je fais aussi ».

Cela veut dire que l'appartenance ethnique constitue un capital social favorisant l'insertion à la coopérative COMAGOA-ROXI.

\section{II.2 Les réseaux de famille ou de parenté}

Les réseaux de familles constituent la deuxième stratégie utilisée par les femmes pour intégrer la coopérative COMAGOA-ROXI. Ainsi, certaines femmes ont pu intégrer la coopérative grâce à ces réseaux de famille. Le lien de parenté avec un membre de la coopérative, et plus spécifiquement la première présidente feue Nanti Lou Rosalie, a permis à ces femmes d'intégrer la coopérative. Cette présidente, qui est la fondatrice de la coopérative, est une caution morale suffisante pour s'insérer dans la coopérative COMAGOAROXI. À cet effet, Z L N, membre de la coopérative, déclare :

"c'est grâce à Nanti Lou Rosalie que je suis rentrée dans coopérative là, je suis la fille de sa grande saur donc elle m'a dit de venir on va travailler ensemble comme moi aussi je vendais vivrier là ».

Ces propos confirment le fait que les réseaux de famille constituent l'un des moyens sûrs pour intégrer la coopérative. Le réseau de famille permet également d'avoir une position honorable, voire très privilégiée, dans les structures associatives. Pierre Bourdieu précise également que certaines personnes arrivent à accéder à des postes puissants par des raccordements sociaux.

Cette déclaration confirme le fait que le réseau de famille dans les associations comme la coopérative s'avèrent être des conditions plus qu'évidentes pour occuper des postes au sein des coopératives. Cependant, les lois de la coopérative stipulent qu' étant toutes membres, elles ont les mêmes droits et peuvent se présenter à tous les postes quel que soient les affinités. Nous constatons que cela semble ne pas être respecté, cela aux dires de T L G, membre de la coopérative :

«Coopérative là, moi aussi je travaille et j'ai commencé avec Nanti donc faut pas ils vont donner toujours à ses parents de sang moi aussi c'est ma sœur Gouro et je veux être présidente aussi »

$\mathrm{Au}$ regard de ces propos, il ressort que l'appartenance à la famille de Nanti Lou dans le cas de COMAGOA-ROXI prédispose à la présidence de la structure. Cela illustre bien les dires de Pierre Bourdieu (1986) qui affirme que le capital social peut être une source d'inégalité. En effet, le fait qu'au sein des groupements associatifs le capital social soit un puissant facteur d'intégration 
peut être une source de conflit pour celle qui ne dit pas son nom, et cela peut fragiliser l'unité du groupe et être source de discordance.

\section{II.3 Le réseau relationnel ou amical}

Au niveau du cadre relationnel, nous pouvons dire que cela intervient indéniablement dans l'insertion au sein des activités économiques. L’insertion et le maintien au sein des groupements associatifs est fonction aussi du réseau de relation que l'individu a à son actif ou que l'individu mobilise. En ce sens, Bourdieu dit que le capital social n'est pas rattaché à l'individu mais permet de rendre existant et de caractériser les relations entre les individus. Il se développe en fonction de trois dimensions : le niveau de confiance sociale, l'existence d'un système légal et l'existence de canaux de communication.

Dans le cadre de COMAGA-ROXI, le niveau de confiance sociale est un moyen d'insertion qui a permis à bon nombre de femmes d'intégrer la coopérative. À ce titre D L F déclare :

« moi avant de venir dans coopérative là je vendais sur même place avec Rosalie et on est devenu camarade, elle me connait elle sait comment je travaille donc quand elle à créer coopérative là elle est venue me voir et m'a dit ma sœur comme j'ai confiance dans toi là viens on va travailler ensemble ».

Ces propos démontrent que le niveau de confiance pour s'insérer est de mise, ce qui veut dire par là que dans la coopérative ne peut pas intégrer une personne qu'elle ne connait pas ; il faudrait que la nouvelle adhérente ait un bon témoignage d'au moins un membre de la coopérative.

\section{Logiques d'intégration}

\section{III.1 Logique pécuniaire}

Il s'avère que les coopératives, étant une entité qui regroupe des personnes exerçant la même activité, ont cette facilité de voir leur activité être financée soit par une cotisation interne effectuée par les membres eux-mêmes, soit par des structures de financement de micro ou macro projet ou simplement par des dons. Aussi, la coopérative octroie des prêts aux membres.

À cet effet une enquêtée affirme :

« moi je suis venue dans coopérative parce que ma camarade avec qui on se débrouille pour vendre un peu de vivrier, son affaire a commencé à devenir grand que pour moi, donc comme demander n'est pas mauvais, j'ai dit Irié. Lou c'est quoi tu fais et puis maintenant je vois que tu as l'argent et ta marchandise la est beaucoup la faut me dire aussi je vais faire. C'est ça elle m'a dit que elle fait coopérative, c'est ça j'ai demandé moi je peux rentrer aussi dans coopérative là, et ma camarade là a dit oui. C'est à cause de ça que moi je suis arrivée dans coopérative là ». 
Les propos de cette enquêtée révèlent que la recherche de gain afin de financer leur activité a été une source de motivation concernant leur insertion au niveau de la coopérative. Pour ces femmes, la coopérative représente une structure de financement qui peut les aider quant au financement de leurs activités et aussi leur procurer un mieux-être. Le but de tout individu exerçant une activité est de voir cette activité fructifier. Cette activité doit donc connaître une croissance qui est synonyme de développement. Cependant, ce développement ne doit pas être à court ni à moyen terme mais un développement à long terme. Comment pérenniser son activité et pouvoir le léguer plus tard à sa descendance ? Telle est la problématique qui a amené certaines femmes à intégrer la coopérative COMAGOA. En effet, exerçant un métier à savoir la vente de vivrier, il leur manquait le capital économique qui allait leur permettre de développer leur activité. Ainsi, leur intégration à la coopérative COMAGOA leur a permis non seulement d'augmenter leur capital économique mais aussi de développer leur commerce. Pour ces enquêtées, la coopérative représente un capital social qu'elles ont mobilisé pour non seulement être propriétaire de parcelles de champs mais aussi pour être pourvoyeuse d'emploi dans la mesure où elles financent des producteurs et offrent du travail, à savoir embaucher des ouvriers dans des plantations. Leur insertion dans la coopérative représente pour elles un facteur non seulement de développement personnel mais aussi un facteur de développement social, car elles se sentent utiles à la société. Cela leur a aussi permis une meilleure organisation de leur activité et aussi de l'envisager sur un long terme. Cet état de fait révèle que la coopérative dans le cadre de leur activité a été un facteur de développement qui leur a permis de se réaliser et d'améliorer leur condition de vie.

\section{III.2 Logique symbolique}

La coopérative est un regroupement d'individus œuvrant pour la même cause. Ici, il s'agit de la vente du vivrier. La vente du vivrier en Côte d'Ivoire est majoritairement attribuée aux femmes de l'ethnie Gouro. Ainsi, la quasitotalité des femmes appartenant à la coopérative COMAGOA sont des femmes Gouro. Cette appartenance a permis à bon nombre de femmes d'intégrer la coopérative COMAGOA car la fondatrice de cette coopérative était une femme Gouro. En cela, une femme dit : «Moi je suis femme Gouro hoo, coopérative là c'est pour ma sour Gouro et nous femmes Gouro on fait vivrier quand on n'est pas allé à l'école, donc comme c'est affaire de Gouro là, j'ai dit que je vais rentrer dedans ».

Ces propos montrent que l'appartenance ethnique est l'une des sources d'intégration au sein de la coopérative COMAGOA. Symboliquement, le groupe ethnique représente pour ces femmes l'appartenance à une famille non pas biologique mais culturelle. Cette appartenance crée un sentiment de 
sécurité et de confiance pour les membres. Certains membres sont aussi parentés à la première présidente de la coopérative, donc ce lien de parenté là a été un facteur d'intégration au sein de cette coopérative. Cette représentation de la coopérative comme un patrimoine peut constituer un obstacle à l'insertion des femmes qui souhaitent l'intégrer, et cela peut constituer également une entrave à la loi de la coopérative qui dit que toute personne exerçant la même activité peut intégrer et bénéficier des mêmes avantages. En cela un membre dit :

«Aujourd'hui pour rentrer dans coopérative là on paye 1500000f, parce ce que nous on a commencé coopérative là, on a travaillé, alors que loi là dit que kan quelqu'un rentre dans coopérative là on a même droit donc c'est normal que nous aussi on augmente prix pour rentrer là. Quelqu'un peut pas venir prendre travail que nous on a fait et puis aujourd'hui on est là là ou bien».

Ces propos révèlent que ces femmes considèrent la coopérative comme étant une entité émanant du fruit de leurs efforts qu'il faut protéger et préserver et non comme une association autonome de personnes volontaires réunies pour satisfaire leurs aspirations et besoins économiques, sociaux et culturels communs au moyen d'une entreprise dont la propriété est collective et où le pouvoir est exercé démocratiquement (Alliance Coopérative International). Ainsi donc, la représentation que ces femmes se font de la coopérative est en porte à faux avec la coopérative qui se veut une structure démocratique, alors qu'on constate que ce principe n'est pas appliqué.

\section{Discussion}

\section{Mobilisation des ressources comme stratégie d'insertion}

La stratégie d'intégration et de maintien dans les activités économiques, particulièrement au sein des groupements associatifs tels que les coopératives, peut se faire de plusieurs manières. Dans le contexte de l'insertion professionnelle des femmes, la vie associative active est présentée comme une association de réseautage et de rentabilité (Helly, 2001). Cet ensemble de réseaux est définit par Pierre Bourdieu comme le capital social. Pour lui, le capital social est l'ensemble des ressources actuelles ou potentielles qui sont à la possession d'un réseau durable de relation plus ou moins institutionnalisées d'interconnaissance et d'inter-reconnaissance (Bourdieu, 1986). De la même manière, au niveau des femmes de la coopérative COMAGOA nous avons pu identifier plusieurs réseaux que les femmes ont pu utiliser pour s'insérer et se maintenir dans l'activité de vente au sein de la coopérative, à savoir les réseaux de famille ou de parenté, le réseau relationnel ou amical ainsi que les liens d'affinités. 


\section{Logique d'intégration}

L'étude révèle trois logiques et stratégies à savoir la logique pécuniaire, la logique de pérennisation de l'activité et la logique symbolique.

On peut donc dire que la quasi-totalité des membres de la coopérative ont intégré la coopérative en vue de développer leur activité et augmenter leur revenu. Ces femmes pour la plupart sont des veuves et ayant en charge des enfants. Dans le souci de pouvoir prendre en charge leur famille, elles ont trouvé nécessaire d'intégrer la coopérative afin de bénéficier des avantages économiques qu'elle offre.

Concernant la pérennisation de l'activité, le but pour tout individu qui exerce une activité économique est de voir cette activité s'accroître. Cette croissance doit être à long terme. Ainsi, les femmes de la coopérative, avec le soutien économique de cette dernière, vont développer leur commerce dans le but de le léguer plus tard aux enfants.

Au niveau symbolique, l'appartenance ethnique est, on peut le dire, source d'intégration au sein de la coopérative COMAGOA. Les femmes de la coopérative considèrent la coopérative comme un patrimoine appartenant aux femmes Gouro. Pour ces femmes il faut la protéger et la préserver.

\section{Conclusion}

Cette étude met en relief les stratégies développées par les femmes pour leur insertion au sein de la structure coopérative. Ces stratégies se manifestent à travers les capitaux que sont le capital social, le capital symbolique et le capital économique. Ces capitaux qui se traduisent par le recours à l'appartenance ethnique, à la famille, aux réseaux relationnels et aux ressources financières. Les stratégies en question permettent aux femmes d'échapper aux barrières pour entrer à la coopérative. Cette insertion à la coopérative permet aux femmes de développer leur activité et de recevoir des financements de la coopérative, étant celle-ci un regroupement de personnes volontaires s'exerçant la même activité pour leur intérêt. La COMAGOAROXI se doit donc de s'ouvrir aux autres femmes exerçant aussi dans le vivrier. Cela leur permettra non seulement de développer et de rendre plus performante la coopérative mais aussi d'étendre leur système d'entraide. Par ailleurs, en plus de l'entraide, les femmes se réfèrent à la coopérative pour leur survie.

\section{References:}

1. Akindes, A. (1986). Contribution à l'étude du secteur informel en Côte d'Ivoire: cas d'une branche d'activité : la vulcanisation, lettre de solagral-stratégies alimentaire, revue $\mathrm{n}^{\circ} 30$ 
2. Akindes, A. (1986). Initiative populaire et alimentation à Abidjan, le secteur informel à Abidjan, lettre de solagral-stratégies alimentaires, revue $n^{\circ} 30$,

3. Ballouard, M-P. (1973). Le rôle de la femme dans le développement économique de la Cote d'Ivoire, du Mali et du Sénégal, Rennes, Thèse.

4. Bassett, T. (1991). Migration et féminisation de l'agriculture dans le Nord de la Cote d'Ivoire, les spectres de Malthus, Paris, Puf.

5. Bassett, T. (1991). Les spectres de malthus : migration et féminisation de l'agriculture dans le nord de la Cote d'Ivoire, Paris Puf.

6. Bazzi-Veil, L. (2000). Situation de la femme en Afrique de l'Ouest et du Centre, Abidjan.

7. Bisilliat, J. (1977). La place de la femme dans le développement: son rôle dans une économie de service non rémunérés, Paris, Puf.

8. BIT. (2002). L'insertion sociale et professionnelle des femmes, Abidjan.

9. Boserup, E. (1983). La Femme face au Développement Économique (sociologie d'aujourd'hui), Paris, Puf.

10. Bourdieu, P. (1986). Le capital social, Paris, Puf.

11. Chazel, F. (2009). Mobilisation des ressources, Paris, Puf.

12. De Beauvoir, S. (1949). Le deuxième sexe, Gallimard éd. tome I et II.

13. Drancourt, N. (2006). L'insertion des jeunes, Paris, Puf, Que sais-je ?.

14. Guérin, P. (1999). La genèse de l'insertion, Paris, Dunod.

15. Havel, J-E. (1971). La condition de la femme, Paris, Armand Colin.

16. Helly, M. (2001). Insertion professionnel des femmes, Puf, Paris.

17. Herz, B. (1989). Comment associer les femmes au développement économique, Paris, Desclée.

18. Hourcade, J. (1992). Pourquoi la femme ?, Paris, desclée / begedis.

19. Kindervatter, S. (1987). Femme travaillons ensemble pour le développement personnel, économique et communautaire: manuel d'activité destiné aux groupes d'éducation et d'action des femmes, Washington.

20. Lilar, S. (1969). Le malentendu du deuxième sexe, Paris, Puf.

21. Meillassoux, C. (1964). Anthropologie économique des Gouro de Côte d'Ivoire: de l'économie de subsistance à l'agriculture commerciale, Paris mouton.

22. Ministère de la famille de la femme et de l'enfant. (2007). Plan National d'Action de la femme, Abidjan.

23. Musitu, L. (2006). La femme congolaise, pilier de l'économie informel en milieu urbain, Puf, Paris.

24. Organisation des Nation Unies. (1996). Commission Economique pour l'Afrique, Etude sur la responsabilité économique des femmes et leur 
rôle dans le développement socio-économique de l'Afrique, CEA, Addis-Abeba.

25. Ouattara, A. (2013). Les femmes du vivrier, ISTC, mensuel $\mathrm{n}^{\circ} 11$, Abidjan.

26. PNUD. (1995). Rapport mondial sur le développement humain, New York.

27. Rapport de synthèse. (2000). Le rôle économique des femmes dans le développement agricole et rural : promotion des activités génératrices de revenus, CTA, Wageningen.

28. Toure, L. (1994). Le mouvement associatif féminin dans la promotion socio-économique de la femme Ivoirienne : le cas de l'AIDF, mémoire de licence IES, Abidjan.

29. UNICEF. (2002). Analyse de la situation de l'enfant et de la femme, Abidjan.

30. Winifred, W. (1986). L'intégration des femmes dans les projets de développement, Puf, Paris. 